\title{
Opportunistic Interference Avoidance Scheduling for Underlay Cognitive Radio Networks
}

\author{
Alexis A. Dowhuszko ${ }^{1}$, Jyri Hämäläinen ${ }^{1}$, and Zhi Ding ${ }^{2}$ \\ ${ }^{1}$ Department of Communications and Networking, Aalto University, P.O. Box 13000, FI-00076 Aalto, Finland \\ ${ }^{2}$ University of California, Davis, California 95616 \\ E-mail: alexis.dowhuszko@aalto.fi, jyri.hamalainen@aalto.fi, zding@ucdavis.edu
}

\begin{abstract}
An opportunistic scheduling scheme that avoids the interference that a primary multi-antenna Base Station (BS) generates in the secondary single-antenna links of an underlay cognitive radio network is proposed. In this scenario, the primary BS applies a pseudo-random sequence of transmit beamforming vectors to serve its users, which is known beforehand at the secondary Receivers (RXs). Combining this information with the estimated values of slow-varying channel gains, the secondary $\mathrm{RXs}$ are able to identify the time instants where the signals from the different transmit antennas of primary BS sum up destructively in reception. Taking advantage of this information, the secondary transmitters are able to delay their transmissions until the interference coming from the primary BS is far from its peak. Closed form expressions for the mean data rate of the secondary link are derived for different SNR regimes. As expected, the interference mitigation capability of the proposed opportunistic scheduling scheme grows as both the SIR and the activity period of secondary links decrease.
\end{abstract}

\section{INTRODUCTION}

Cognitive radio has been introduced as a promising technology to improve the use of radio spectrum, a precious natural resource that is not always utilized efficiently [1]. In a cognitive radio system, secondary (cognitive) transmissions are activated along with primary (licensed) transmissions, in such a way that primary transmissions are not significantly affected. There are two well-known spectrum access models to protect primary Receivers (RXs), namely Opportunistic Spectrum Access (OSA) and Concurrent Spectrum Access (CSA) [2]. In OSA model, also known as interweaved cognitive radio [3], a secondary Transmitter (TX) utilizes the spectrum when it is not being occupied by a primary transmission. In CSA model, also known as underlay cognitive radio [3], the power spectral mask of secondary transmissions is enforced to keep the interference towards primary RXs under control. The CSA model has many practical applications, and can be used to describe the interference scenario that takes place in Device-to-Device (D2D) communications underlaying macrocellular networks [4], [5].

Device-to-device communication paradigm has been recently introduced within 3GPP for general Proximity Services (ProSe), and first steps in LTE standardization have been taken to integrate D2D communications into conventional networks [6]. Though D2D scenarios are usually related to direct user-to-user communication in public safety context and social networking applications, Machine-to-Machine (M2M) communications also provide a very attractive application area for D2D. Actually, M2M services are expected to be one of the key drivers for traffic growth in cellular networks, with tens of billions connected devices by year 2020 [7], [8]. Two scenarios have been identified in [9] for M2M communications, involving the communication between M2M devices and server, and the direct communication between M2M devices. Though the latter scenario is closely related to D2D communications, the main difference is that direct M2M services are expected to take place between nomadic/static devices, and with infrequent and delay tolerant data transmissions [10], [11].

In this paper we consider a large number of direct M2M links and macro Base Stations (BSs) operating in the same frequency band. This co-channel deployment is equivalent to the CSA model for cognitive radio networks [2], [5], where the interference from secondary M2M TXs to primary macro RXs is controlled using low transmission powers. To tackle the interference from the primary macro BS, an opportunistic communication approach that exploits the delay tolerant nature of M2M data is implemented. That is, secondary transmissions are scheduled when the signals that arrive from the different antennas of the primary macro BS are summed up destructively at the secondary M2M RX. To induce the variation of wireless channel gains in a proper time scale, we assume that the primary macro BS applies a pseudo-random sequence of Transmit Beamforming (TBF) vectors that is known beforehand at the secondary M2M RXs. This enables effective prediction of feasible transmission times, demanding low-rate feedback overhead and making it possible to switch to low-power consumption modes for the rest of the time.

The rest of the paper is organized as follows: Section II introduces the system model and discusses the principles of opportunistic interference-aware scheduling in secondary M2M links. Section III derives closed form formulas to characterize the interference mitigation capabilities of the proposed opportunistic approach, while Section IV presents the performance results and validates the analysis with numerical simulations. Finally, conclusions are drawn in Section V.

\section{SySTEM MODEL}

The general layout of our underlay cognitive radio network is illustrated in Fig. 1. The network contains a fixed primary BS serving a given number of primary Mobile Stations (MSs), and a large number of secondary links that coexist with the primary system using the same spectral resources. The primary BS is equipped with $M$ transmit antennas, while the primary 




Fig. 1. The underlay cognitive radio network comprises a primary BS with $M$ transmit antennas, and a large number of primary MSs and secondary M2M links equipped with a single antenna. The primary BS applies pseudo-random TBF vectors to serve its associated MSs. The secondary RXs know the sequence of $\mathrm{TBF}$ vectors at the primary $\mathrm{BS}$, and inform to the secondary TXs those time instants with low co-channel interference (red dashed lines).

MSs and both secondary TXs and RXs are equipped with a single antenna. The co-channel interference that the secondary links introduce in the primary system is kept under control restricting the maximum transmit power that the secondary TXs are allowed to use. As a consequence, secondary transmissions do not affect the performance of the primary system considerably. The main goal of the paper is to improve the data rate performance of the secondary links, assuming that the primary BS is active all the time, and that the secondary links do not have to transmit data continuously.

\section{A. Signal model for the secondary link}

Consider that a generic secondary link $k$ is laid in the coverage area of the primary BS. Then, its received signal at Transmission Time Instant (TTI) $i$ attains the form

$$
r_{k}[i]=\underbrace{h_{k, k} s_{k}}_{\text {Desired signal }}+\underbrace{\left(\mathbf{h}_{p, k} \cdot \widetilde{\mathbf{w}}[i]\right) s_{p}}_{\text {Interference }}+\underbrace{n_{k}}_{\text {Noise }},
$$

where $h_{k, k}$ is the direct channel gain in the secondary link $k$, $\mathbf{h}_{p, k} \in \mathbb{C}^{1 \times M}$ is the vector that contains the complex channel gains from primary $\mathrm{BS}$ antennas to secondary $\mathrm{RX} k$, $\widetilde{\mathbf{w}}[i] \in \mathbb{C}^{1 \times M}$ is the random $\mathrm{TBF}$ vector that primary BS applies at time instant $i, s_{k}$ and $s_{p}$ are the transmitted complex symbols of secondary TX $k$ and primary BS, respectively, and $n_{k}$ is zero-mean complex Gaussian noise with power $P_{N}$. In case of rich scattering and flat fading, elements $h_{k, m}=\left|h_{k, m}\right| e^{j \psi_{k, m}}$ of channel gain vector $\mathbf{h}_{p, k}=\left(h_{k, 1} \ldots h_{k, M}\right)$ are described by zero-mean circularly symmetric complex Gaussian Random Variables (RVs). That is, module $\left|h_{k, m}\right|$ is Rayleigh distributed with unitary second raw moment, and argument $\psi_{k, m}$ is uniformly distributed in the interval $(-\pi, \pi]$.

\section{B. Interfence-aware scheduling}

To increase the achievable data rate in the secondary links, transmissions should be scheduled in those TTIs where the instantaneous-to-peak data rate ratio surpass a given threshold. Scheduling strategy can be simplified replacing the data rates with received SINR values, i.e.,

$$
\frac{\log _{e}\left(1+\widetilde{\gamma}_{k}[i]\right)}{\log _{e}\left(1+\widehat{\gamma}_{k}\right)} \approx \frac{\widetilde{\gamma}_{k}[i]}{\widehat{\gamma}_{k}}
$$

where

$$
\widetilde{\gamma}_{k}[i]=\frac{\bar{\gamma}_{k, k} g_{k, k}}{\bar{\gamma}_{p, k} \widetilde{g}_{p, k}[i]+1}
$$

and $\widehat{\gamma}_{k}$ represent the instantaneous and peak SINR at the secondary RX, respectively. In (3),

$$
\bar{\gamma}_{k, k}=\frac{P_{\mathrm{tx}, k}}{L_{k, k} P_{N}}, \quad g_{k, k}=\left|h_{k, k}\right|^{2},
$$

are the mean received SNR and the instantaneous channel power gain between secondary TX and RX, while

$$
\bar{\gamma}_{p, k}=\frac{P_{\mathrm{tx}, p}}{L_{p, k} P_{N}}, \quad \widetilde{g}_{p, k}[i]=\left|\mathbf{h}_{p, k} \cdot \widetilde{\mathbf{w}}[i]\right|^{2},
$$

represent the mean received SNR and the equivalent post-TBF channel power gain from the primary BS to the secondary RX. In (4), $P_{\mathrm{tx}, k}$ and $L_{k, k}$ are the transmit power and the path loss attenuation in the secondary link. Similarly, in (5), $P_{\mathrm{tx}, p}$ and $L_{p, k}$ represent the transmit power and the path loss attenuation of the link between primary BS and secondary RX. It is important to highlight that approximation (2) becomes particularly good at low SINR regimes.

Peak SINR $\widehat{\gamma}_{k}$ takes place when the interference coming from the primary BS is low. Low interference at secondary $\mathrm{RX} k$ is experienced when the random TBF vector $\widetilde{\mathbf{w}}[i]$ is far enough from the beamforming configuration

$$
\widehat{\mathbf{w}}_{k}=\arg \max _{\mathbf{w} \in \mathcal{W}}\left|\mathbf{h}_{p, k} \cdot \mathbf{w}\right|^{2},
$$

where $\mathcal{W}$ represents the TBF codebook used at primary BS. So, performance indicator (2) can be replaced by projection

$$
\kappa_{k}[i]=\left|\widehat{\mathbf{w}}_{k} \cdot \widetilde{\mathbf{w}}_{k}[i]\right|^{2},
$$

and transmission in the secondary link can be scheduled when $\kappa_{k}[i]$ lies below a properly selected threshold (which guarantees a desired period of activity in the secondary link).

\section{Codebook-based random beamforming scheme}

In a conventional Random Beamforming (RBF) scheme [12], both amplitudes and phases of the signals of each transmit antenna are varied over time continuously. Overall channel tracking in this situation is done via a common pilot signal, which is broadcast in downlink and affected by a random TBF vector that should vary slowly enough to enable an accurate estimation of instantaneous channel condition in reception. In this situation, the RX needs to update the estimation of the overall channel gain every time the random TBF vector changes (i.e., every $T_{\mathrm{tti}}$ seconds). This represents an excessive use of resources, particularly for 
battery-driven nodes with fixed locations, deployed in mobile communication environments with low mobility scatterers.

To cope with this problem, we take advantage of the large coherence times (i.e., $T_{\text {coh }}$ ) of the channel to design a more practical codebook-based RBF scheme. That is, instead of tracking in reception fast varying overall channel gains $\widetilde{g}_{p, k}[i]$, we consider that each secondary $\mathrm{RX} k$ first keeps track of each slow varying individual channel gain from channel gain vector $\mathbf{h}_{p, k}$. After that, with the aid of (6), the $\mathrm{RX}$ determines the element $\widehat{\mathbf{w}}_{k} \in \mathcal{W}$ that is closest to the beamforming configuration. Finally, based on the a priori knowledge of the sequence of pseudo-random TBF vectors $\left\{\widetilde{\mathbf{w}}[i]: i=1, \ldots,\left\lfloor T_{\mathrm{coh}} / T_{\mathrm{tti}}\right\rfloor\right\}$, the secondary $\mathrm{RX}$ informs to the secondary TX the indexes of the TTIs where projection indicator (7) will be below a predefined threshold $\kappa_{\text {th }}$. With this precise information, both secondary TX and RX will know in advance the time instants where transmission will be scheduled in the secondary link, being in active state at those moments and having the chance to switch to a low power consumption mode to save energy the rest of the time.

The interference avoidance scheme that is proposed in this paper assumes that pseudo-random TBF vector $\widetilde{\mathbf{w}}[i]$ is known at secondary RXs for all time instants $i$. Since valid TBF vectors are forced to belong to a finite-size TBF codebook $\mathcal{W}$, this assumption is valid if the sequence of TBF vectors is either explicitly informed from primary BS to secondary RXs, or stored in the memory of secondary RXs beforehand. In the former case, long-term signaling is needed in the initialization of the interference avoidance scheme. In the latter case, secondary RXs need to synchronize to the pseudo-random sequence that the primary BS applies.

\section{Feedback information characterization}

Amplitude randomization leads to poor PA efficiency [12]. Therefore, we consider that the power per transmit antenna is kept constant at the primary BS, and only the phases of the signals applied in the different transmit antennas are randomly changed. Phases of individual channel gains are uniformly distributed in $(-\pi, \pi]$. Therefore, a uniform phase quantizer is used to define the elements $\mathbf{w}=\left(w_{1} \ldots w_{M}\right)$ of TBF codebook $\mathcal{W}$ as follows:

$$
w_{m}=\frac{1}{\sqrt{M}} e^{j \frac{(2 n-1) \pi}{2^{N}}} \quad n=1, \ldots, 2^{N},
$$

where $N$ is the number of cophasing bits per transmit antennas, which generate $2^{N}$ different quantized cophasing values that randomize the phases of transmit antennas independently.

Let $\mathcal{K}$ be the set of all possible discrete values that projection indicator $\kappa_{k}[i]$ can take when both $\widehat{\mathbf{w}}_{k}$ and $\widetilde{\mathbf{w}}[i]$ belong to TBF codebook $\mathcal{W}$. The elements of set $\mathcal{K}$ and their corresponding probability of occurrence were studied in [13] for $N=1,2$. Unfortunately, it is not easy to obtain general closed form expressions when $N \geq 3$. To cope with this limitation, in this paper we use a continuous approximation that works asymptotically tight as $M$ and $N$ grow. This approximation is based on previously derived results on Random Vector
Quantization (RVQ) beamforming [14], [15], and claims that the Cumulative Distribution Function (CDF) of (7) verifies

$$
F_{\kappa_{k}}(x) \approx 1-(1-x)^{M-1} \quad 0 \leq x \leq 1 .
$$

Note that to apply this approximation, we have considered that $\widehat{\mathbf{w}}_{k}$ and $\widetilde{\mathbf{w}}[i]$ are independent. The level of fitness of approximation (9) will be studied in detail in Section IV.

\section{RATE PERFORMANCE ANALYSIS}

To compute the mean data rate of a secondary link when proposed opportunistic scheduling scheme that avoids interference based on projection (7) is implemented, the CDF of the received SINR at secondary RX needs to be determined. To achieve this goal, we first model the stochastic behavior of the interference that primary BS generates on secondary RX.

\section{A. Probability distribution function for received interference}

In this section we determine the Probability Distribution Function (PDF) of the normalized overall interference $\widetilde{g}_{p, k}[i]=\left|\mathbf{h}_{p, k} \cdot \widetilde{\mathbf{w}}[i]\right|^{2}$, when a transmission is scheduled in the secondary link. Let us define $\Delta_{k, m}[i]=\left(\psi_{k, m}+\widetilde{\phi}_{m}[i]\right)$, where $\widetilde{\phi}_{m}[i]$ is the random phase that primary BS applies in antenna $m$ at time instant $i$. Then, it is possible to expand

$$
\begin{aligned}
\widetilde{g}_{p, k}[i] & =\left(\sum_{m=1}^{M} \frac{\left|h_{k, m}\right|}{\sqrt{M}} e^{j \Delta_{k, m}[i]}\right)\left(\sum_{m=1}^{M} \frac{\left|h_{k, m}\right|}{\sqrt{M}} e^{j \Delta_{k, m}[i]}\right)^{*} \\
& =\frac{1}{M} \sum_{m=1}^{M}\left|h_{k, m}\right|^{2}+\frac{2}{M} \sum_{m_{1}=1}^{M} \sum_{m_{2} \neq m_{1}}\left|h_{k, m_{1}}\right| \\
& \times\left|h_{k, m_{2}}\right| \operatorname{Re}\left\{e^{j\left(\Delta_{k, m_{1}}[i]-\Delta_{k, m_{2}}[i]\right)}\right\}
\end{aligned}
$$

Applying the expectation in the previous expression, it is possible to observe that

$$
\begin{aligned}
\mathbb{E}\left\{\widetilde{g}_{p, k}\right\} & =\frac{1}{M} \sum_{m=1}^{M} \mathbb{E}\left\{\left|h_{k, m}\right|^{2}\right\} \\
& +\frac{2}{M} \sum_{m_{1}=1}^{M} \sum_{m_{2} \neq m_{1}} \mathbb{E}\left\{\left|h_{k, m_{1}}\right|\right\} \mathbb{E}\left\{\left|h_{k, m_{2}}\right|\right\} \\
& \times \mathbb{E}\left\{\operatorname{Re}\left\{e^{j\left(\Delta_{k, m_{1}}-\Delta_{k, m_{2}}\right)}\right\}\right\} \\
& =\frac{1}{M} \sum_{m=1}^{M} \mathbb{E}\left\{|h|^{2}\right\}+\frac{2}{M} \mathbb{E}^{2}\{|h|\} \\
& \times \sum_{m_{1}=1}^{M} \sum_{m_{2} \neq m_{1}} \mathbb{E}\left\{\operatorname{Re}\left\{e^{j\left(\Delta_{k, m_{1}}-\Delta_{k, m_{2}}\right)}\right\}\right\} \\
& =\mathbb{E}\left\{|h|^{2}\right\}+\mathbb{E}\{|h|\}\left(M \mathbb{E}\left\{\kappa_{k}\right\}-1\right) \\
& =1+\frac{\pi}{4}\left(M \mathbb{E}\left\{\kappa_{k}\right\}-1\right) .
\end{aligned}
$$

Based on this formula, there is always a positive lower bound for the interference power at the secondary $\mathrm{RX}$ if primary BS randomizes only the phases of transmit antennas; that is, $\mathbb{E}\left\{\widetilde{g}_{p, k}\right\} \rightarrow(1-\pi / 4)$ when $\mathbb{E}\left\{\kappa_{k}\right\} \rightarrow 0$. For further interference avoidance capabilities, the TBF codebook $\mathcal{W}$ that is used at the primary BS should also randomize the power that is applied in each transmit antenna. 
TABLE I. Fading figure of normalized interference under different working regimes.

\begin{tabular}{|c|c||c|c|c|c|c||}
\hline \multicolumn{2}{|c|}{ Period of Activity } & $20 \%$ & $40 \%$ & $60 \%$ & $80 \%$ & $100 \%$ \\
\hline \multirow{3}{*}{$M=4$} & $N=2$ & 0.90 & 0.90 & 0.97 & 1.04 & 1.00 \\
\cline { 2 - 7 } & $N=3$ & 0.89 & 0.90 & 0.99 & 1.07 & 1.00 \\
\cline { 2 - 7 } & $N=4$ & 0.86 & 0.94 & 1.02 & 1.08 & 1.00 \\
\hline \multirow{3}{*}{$M=8$} & $N=2$ & 0.95 & 0.98 & 1.02 & 1.09 & 1.00 \\
\cline { 2 - 7 } & $N=3$ & 0.94 & 1.00 & 1.08 & 1.15 & 1.00 \\
\cline { 2 - 7 } & $N=4$ & 0.94 & 1.03 & 1.11 & 1.17 & 1.00 \\
\hline
\end{tabular}

Transmission in secondary link $k$ takes place whenever $\kappa_{k}[i] \leq \kappa_{\mathrm{th}}$. In this situation, the conditional expected value of $\kappa_{k}$ when transmission is scheduled attains the form

$$
\begin{aligned}
\mathbb{E}\left\{\kappa_{k} \mid \mathrm{tx}\right\} & =\int_{0}^{\kappa_{\mathrm{th}}} x \frac{f_{\kappa}(x)}{F_{\kappa}\left(\kappa_{\mathrm{th}}\right)} d x \\
& =\kappa_{\mathrm{th}}-\frac{\int_{0}^{\kappa_{\mathrm{th}}} F_{\kappa}(x) d x}{F_{\kappa}\left(\kappa_{\mathrm{th}}\right)} \\
& =\kappa_{\mathrm{th}}-\frac{\kappa_{\mathrm{th}}-[1 / M]\left[1-\left(1-\kappa_{\mathrm{th}}\right)^{M}\right]}{1-\left(1-\kappa_{\mathrm{th}}\right)^{M-1}} .
\end{aligned}
$$

Plugging (12) in (11), the conditional expectation of the normalized interference $\mathbb{E}\left\{\widetilde{g}_{p, k} \mid \mathrm{tx}\right\}$ when transmission is scheduled is determined. Following a similar procedure, a closed form expression for the conditional second raw moment $\mathbb{E}\left\{\widetilde{g}_{p, k}^{2} \mid \mathrm{tx}\right\}$ can also be found. With these two statistics, the fading figure for the normalized interference that secondary $\mathrm{RX} k$ experiences reception, i.e.,

$$
\mathcal{F}_{\widetilde{g}_{p, k} \mid \mathrm{tx}}=\frac{\mathbb{E}^{2}\left\{\widetilde{g}_{p, k} \mid \mathrm{tx}\right\}}{\operatorname{Var}\left\{\widetilde{g}_{p, k} \mid \mathrm{tx}\right\}}=\frac{\mathbb{E}^{2}\left\{\widetilde{g}_{p, k} \mid \mathrm{tx}\right\}}{\mathbb{E}\left\{\widetilde{g}_{p, k}^{2} \mid \mathrm{tx}\right\}-\mathbb{E}^{2}\left\{\widetilde{g}_{p, k} \mid \mathrm{tx}\right\}},
$$

can be determined [16]. Based the fact that fading figure takes values close to unity for different periods of activity $\operatorname{Pr}\left\{\kappa_{k}[i] \leq \kappa_{\text {th }}\right\}$, see Table I, it is possible to claim that the PDF of interference $Y=\bar{\gamma}_{p, k} \widetilde{g}_{p, k \mid \mathrm{tx}}$ can be approximated as

$$
f_{Y}(y) \approx \lambda_{y} e^{-\lambda_{y} y} \quad y \geq 0,
$$

with $\lambda_{y}{ }^{-1}=\mathbb{E}\{Y\}=\bar{\gamma}_{p, k} \mathbb{E}\left\{\widetilde{g}_{p, k} \mid \mathrm{tx}\right\}$. With this information, we are now able to estimate the CDF for the received SINR.

\section{B. Cumulative distribution function for received SINR}

To model the stochastic behavior of the received SINR at a secondary link $k$, we consider RV

$$
Z=\frac{X}{1+Y}
$$

assuming that RVs $X$ and $Y$ are both independent exponentially distributed RVs with mean

$$
\mathbb{E}\{X\}=\bar{\gamma}_{k, k}, \quad \mathbb{E}\{Y\}=\bar{\gamma}_{p, k} \mathbb{E}\left\{\widetilde{g}_{p, k} \mid \mathrm{tx}\right\},
$$

respectively [17]. Then, with the aid of results of [18], it is possible to show that the CDF of RV $Z$ is of the form

$$
\begin{aligned}
F_{Z}(z) & =\int_{0}^{\infty} F_{X}[z(t+1)] f_{Y}(t) d t \\
& =1-\frac{e^{-\frac{z}{\mathbb{E}\{X\}}}}{\mathbb{E}\{Y\}} \int_{0}^{\infty} e^{-t\left(\frac{z}{\mathbb{E}\{X\}}+\frac{1}{\mathbb{E}\{Y\}}\right)} d t \\
& =1-\frac{\mathbb{E}\{X\} e^{-\frac{z}{\mathbb{E}\{X\}}}}{\mathbb{E}\{Y\} z+\mathbb{E}\{X\}} \quad z \geq 0 .
\end{aligned}
$$

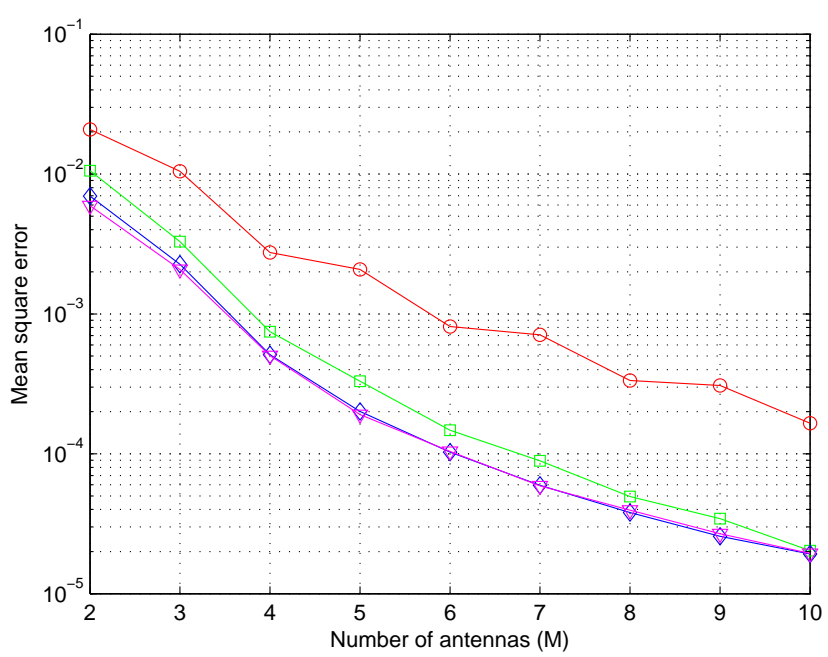

Fig. 2. Mean square error in proposed approximation for cumulative distribution function of projection $\kappa_{k}[i]$. Primary BS applies pseudo-random TBF vectors from codebook designed for $M$ transmit antennas and $N$ phase bits per antenna. Red line (circles): $N=2$. Green line (squares): $N=3$. Blue line (diamonds): $N=4$. Magenta line (triangles): $N=5$.

Plugging expectations (16) in the previous formula, the CDF for received SINR is obtained.

\section{Mean data rate in the secondary link}

By definition, the mean data rate in the secondary link is

$$
R_{k}=\int_{0}^{\infty} \log _{2}(1+\gamma) f_{\widetilde{\gamma}_{k} \mid \mathrm{tx}}(\gamma) d \gamma
$$

or equivalently,

$$
\begin{aligned}
R_{k} & =\log _{2}(e)\left\{\left.\left[\log _{e}(1+\gamma) F_{\widetilde{\gamma}_{k} \mid \mathrm{tx}}(\gamma)\right]\right|_{0} ^{\infty}\right. \\
& \left.-\int_{0}^{\infty} \frac{F_{\widetilde{\gamma}_{k} \mid \mathrm{tx}}(\gamma)}{(1+\gamma)} d \gamma\right\}
\end{aligned}
$$

applying integration by parts. Combining the previous expression with the CDF derived in (17),

$$
\begin{aligned}
R_{k} & =\log _{2}(e) \alpha \int_{0}^{\infty} \frac{e^{-\mu \gamma}}{(\gamma+\alpha)(\gamma+1)} d \gamma \\
& =\log _{2}(e) \frac{\alpha}{(1-\alpha)}\left\{\int_{0}^{\infty} \frac{e^{-\mu \gamma}}{(\gamma+\alpha)} d \gamma-\int_{0}^{\infty} \frac{e^{-\mu \gamma}}{(\gamma+1)} d \gamma\right\} \\
& =\log _{2}(e) \frac{\alpha}{(1-\alpha)}\left\{e^{\alpha \mu} E_{1}(\alpha \mu)-e^{\mu} E_{1}(\mu)\right\}
\end{aligned}
$$

is obtained with the aid of definite integral formula (3.352.4) of [19], where

$$
\alpha=\frac{\mathbb{E}\{X\}}{\mathbb{E}\{Y\}}=\frac{\bar{\gamma}_{k, k}}{\bar{\gamma}_{p, k} \mathbb{E}\left\{\widetilde{g}_{p, k} \mid \mathrm{tx}\right\}}, \quad \mu=\frac{1}{\mathbb{E}\{X\}}=\frac{1}{\bar{\gamma}_{k, k}},
$$

and $E_{1}(x)=\int_{1}^{\infty} e^{-x t} / t d t$ is the exponential integral function [20]. 




(a) $\bar{\gamma}_{k, k}=0 \mathrm{~dB}$



(b) $\bar{\gamma}_{k, k}=10 \mathrm{~dB}$

Fig. 3. Mean data rate in secondary link $k$ as function of the period of activity. Primary BS applies pseudo-random TBF vectors from codebook designed for $M=4$ transmit antennas $N=3$ phase bits per antenna. Transmission in the secondary link is scheduled whenever projection $\kappa_{k}[i]$ surpass pre-defined threshold $\kappa_{\text {th }}$ (for target period of activity). Red dashed lines (circles): $\bar{\gamma}_{p, k}=\bar{\gamma}_{k, k}$. Green dashed lines (squares): $\bar{\gamma}_{p, k}=\bar{\gamma}_{k, k}+5$ dB. Blue dashed lines (diamonds): $\bar{\gamma}_{p, k}=\bar{\gamma}_{k, k}+10 \mathrm{~dB}$. Solid black lines: Simulated values.

\section{Performance Results}

For illustrative purposes, Fig. 2 shows the level of fitness that approximation (9) provides when modeling the actual stochastic behavior of $\mathrm{RV} \kappa_{k}[i]$. The level of fitness is measured in terms of the Mean Square Error (MSE), i.e.,

$\operatorname{MSE}=\int_{0}^{1}|\epsilon(x)|^{2} d x=\int_{0}^{1}\left|F_{\kappa}(x)-\left[1-(1-x)^{M-1}\right]\right|^{2} d x$,

and is presented for different values of $M$ and $N$. As expected, the accuracy of the proposed approximation increases as both $M$ and $N$ grow. This is because the number of discrete values that $\kappa_{k}[i]$ can take increases in both cases, making the actual staircase behavior of $F_{\kappa_{k}}(x)$ become more and more similar to the one of the continuous approximation presented in (9).

Figure 3 shows the mean data rate that can be achieved in the secondary link when the TBF codebook $\mathcal{W}$ of the primary $\mathrm{BS}$ is designed for $M=4$ transmit antennas and $N=3$ phase bits per antenna, according to the guidelines presented in Section II-D. The sequence of pseudo-random TBF vectors is known in advance at the secondary RX, and is used to inform the secondary TX about the time instants of low interference coming from the primary BS. Figure $3 \mathrm{a}$ assumes that the mean SNR of secondary link is $\bar{\gamma}_{k, k}=0 \mathrm{~dB}$, while Fig. $3 \mathrm{~b}$ considers $\bar{\gamma}_{k, k}=10 \mathrm{~dB}$. Note that in an underlay cognitive radio network, the former case is representative of a secondary link that is placed in the outer area of a primary cell, while the latter case corresponds to a secondary link that is situated in the inner part. Dashed lines present the estimated values for the mean data rates for different mean interference powers coming from the primary BS: $\bar{\gamma}_{p, k}=\bar{\gamma}_{k, k}$ (circles), $\bar{\gamma}_{p, k}=\bar{\gamma}_{k, k}+5 \mathrm{~dB}$ (squares) and $\bar{\gamma}_{p, k}=\bar{\gamma}_{k, k}+10 \mathrm{~dB}$ (diamonds). In all cases, simulated values are presented with solid lines, and are included to validate the closed form approximation derived in Section III-C.

As expected, the interference avoidance capability of the proposed opportunistic scheduling scheme increases as the SIR of the secondary link decreases. This gain becomes even larger when the SNR of the secondary link grows. The lower is the period of activity that is required in the secondary link, the larger is the gain that an opportunistic interference-aware scheduling scheme is able to reap. The latter comparison is done with respect to the scheduling scheme that is not aware of interference, and whose mean data rate is equivalent to the one obtained when the period of activity of the proposed opportunistic scheduling scheme is $100 \%$.

\section{CONCLusions}

Interference-aware scheduling provides a good alternative to avoid the peaks of co-channel interference that a primary BS generates in the secondary RXs of an underlay cognitive radio network. The proposed opportunistic scheduling scheme has a lot of applications in contemporary communication scenarios and, among others, can be used to improve the performance of direct M2M communications underlaying macrocellular networks. Exploiting the infrequent and delay tolerant nature of M2M data services, secondary transmissions were delayed until the interference coming from the primary macro BS was low enough. To induce larger variations of the instantaneous interference power in a proper time scale, a pseudo-random sequence of TBF vectors was applied at the primary macro BS. Knowing the sequence in advance, the secondary M2M links were able to determine feasible time instants to transmit data. With the implementation of interference avoidance scheduling, notable gains in terms of mean data rate were observed in the secondary M2M links. As expected, this gain depended not 
only on the SIR situation of the secondary links, but also on the activity period of secondary M2M data services.

\section{ACKNOWLEDGMENT}

This material is based on work supported by the Academy of Finland (grants 257708 and 254299).

\section{REFERENCES}

[1] S. Haykin, "Cognitive radio: brain-empowered wireless communications," IEEE J. Sel. Areas Commun., vol. 23, no. 2, pp. 201-220, Feb. 2005.

[2] Y.-C. Liang, K.-C. Chen, G. Li, and P. Mahonen, "Cognitive radio networking and communications: an overview," IEEE Trans. Veh. Tech., vol. 60, no. 7, pp. 3386-3407, Sep. 2011.

[3] S. Srinivasa and S. A. Jafar, "The throughput potential of cognitive radio: A theoretical perspective," IEEE. Commun. Mag., vol. 45, no. 5, pp. 73-79, May 2007.

[4] L. Lei, Z. Zhong, C. Lin, and X. Shen, "Operator controlled deviceto-device communications in LTE-advanced networks," IEEE Wireless Commun., vol. 19, no. 3, pp. 96-104, Jun. 2012.

[5] Y. Pei and Y.-C. Liang, "Resource allocation for device-to-device communications overlaying two-way cellular networks," IEEE Trans. Wireless Commun., vol. 12, no. 7, pp. 3611-3621, Jul. 2013.

[6] 3GPP, "Feasibility study for proximity services (ProSe) (Rel. 12)," 3GPP technical report, TR 22.803, Ver. 12.1.0, Mar. 2013.

[7] D. Evans, "The internet of things - How the next evolution of the Internet is changing everything," Cisco whitepaper, Apr. 2011.

[8] M. Hatton, "The global M2M market in 2013," Machina research whitepaper, Jan. 2013.

[9] 3GPP, "Service requirements for Machine-Type Communications (MTC) - stage 1," 3GPP technical report, TR 22.368, Ver. 12.2.0, Jun. 2011.

[10] IEEE 802.16p-10/0005, "Machine-to-Machine (M2M) communications technical report," IEEE 802.16 Broadband Wireless Access Working Group, Nov. 2010.
[11] IEEE 802.16p-11/0014, "IEEE 802.16p Machine-to-Machine (M2M) Evaluation Methodology Document (EMD)," IEEE 802.16 Broadband Wireless Access Working Group, May 2010.

[12] P. Viswanath, D. N. C. Tse, and R. Laroia, "Opportunistic beamforming using dumb antennas," IEEE Trans. Inf. Theory, vol. 48, no. 6, pp. 12771294, Jun. 2002.

[13] A. A. Dowhuszko, G. Corral-Briones, J. Hämäläinen, and R. Wichman, "On the analysis and design of practical quantization for opportunistic beamforming," in Proc. IEEE Int. Conf. on Commun., May 2008, pp. 5133-5139.

[14] C. K. Au-Yeung and D. J. Love, "On the performance of random vector quantization limited feedback beamforming in a MISO system," IEEE Trans. Wireless Commun., vol. 6, no. 2, pp. 458-462, Feb. 2007.

[15] A. A. Dowhuszko, J. Hämäläinen, A. R. Elsherif, and Z. Ding, "Performance of transmit beamforming for interference mitigation with random codebooks," in Proc. Int. Conf. Cognitive Radio Oriented Wireless Networks, Jul. 2013, pp. 190-195.

[16] A. A. Dowhuszko, G. Corral-Briones, J. Hämäläinen, and R. Wichman, "On throughput-fairness tradeoff in virtual MIMO systems with limited feedback," EURASIP Journal on Wireless Commun. and Networking, vol. 2009, pp. 1-17, 2009.

[17] A. A. Dowhuszko, M. Husso, J. Li, J. Hämäläinen, and Z. Zheng, "Performance of practical transmit beamforming methods for interference suppression in closed-access femtocells," in Proc. Future Network and MobileSummit (Workshop on Broadband Femtocell Networks), Jun. 2011, pp. 1-12.

[18] A. Papoulis, Probability, random variables, and stochastic processes, 3rd ed. McGraw-Hill International Editions, 1991.

[19] I. S. Gradshteyn and I. M. Ryzhik, Table of Integrals, Series, and Products, 7th ed. Academic Press, New York, 2007.

[20] M. Abramowitz and I. A. Stegun, Handbook of Mathematical Functions. National Bureau of Standards, Washington, DC, 1972. 